10

\title{
LOS ROSTROS DE LA UNIVERSIDAD: ACADEMIA, CULTURA Y POLÍTICA
} THE FACES OF UNIVERSITY: ACADEMY, CULTURE AND POLITICS

Fabiana Alonso

\begin{abstract}
fabianaalonso11@hotmail.com /
Profesora adjunta ordinaria e investigadora del Departamento de Historia de la Facultad de Humanidades y Ciencias (Universidad Nacional del Litoral) y profesora asociada ordinaria de la Licenciatura en Historia de la Facultad de Humanidades, Artes y Ciencias Sociales (Universidad Autónoma de Entre Ríos).
\end{abstract}

Fecha de recepción

$>07 / 07 / 21$
Fecha de aceptación

$>25 / 08 / 21$ 


\section{RESUMEN}

El presente artículo da cuenta de un proyecto de investigación cuyo objeto de estudio se recorta en la Universidad Nacional del Litoral atendiendo a sus relaciones con el ámbito socio-político local y regional. Dichas relaciones expresan un vínculo instituyente en un doble sentido: las interpelaciones de la sociedad a la institución universitaria y las contribuciones de esta a la constitución de la sociedad de la que es parte. Desde esta perspectiva se pretende identificar e indagar las zonas de intersección entre la producción académica y la intervención cultural y política, contemplando las complejas relaciones de la institución universitaria con los cambiantes escenarios políticos de la Argentina del siglo XX. Para desarrollar la investigación se recuperan aportes de la historia política renovada, de la historia conceptual, de la historia intelectual y del análisis del discurso.

\section{ABSTRACT}

This article gives an account about a research which defines its object on the Universidad Nacional del Litoral, based on its relations with the local and socio-political sphere. This relationship expresses an instituting link in a double sense: society challenges university institution and it, in turn, contributes to the constitution of society of which it is a part. This perspective allows to identify and investigate the intersection areas between academic production and cultural and political intervention, considering as well the complex relationship between university and the changing political scenarios of the 20th century in Argentina. In order to develop the research, contributions from renewd political history, history of concepts, intelectual history and discourse analysis are recovered.

\section{PALABRAS CLAVE}

$>$ agentes universitarios

$>$ esfera pública

$>$ campo cultural

\section{KEYWORDS}

$>$ university agents

$>$ public sphere

$>$ cultural field 


\section{INTRODUCCIÓN}

El proyecto de investigación «Academia, cultura y política en espacios regionales. La Universidad Nacional en el siglo XX» recorta su objeto de estudio sobre la institución mencionada (en adelante, UNL) y sus relaciones con el ámbito socio-político local y regional. ${ }^{1}$ Este señalamiento se sostiene en la hipótesis que considera a la UNL como un agente en la constitución del campo político y cultural de la ciudad de Santa Fe y la región. En este sentido, se plantea que la universidad tiene dos rostros, interrelacionados en la práctica y, a la vez, pasibles de ser diferenciados para su estudio. Como institución de ciencia y enseñanza, uno de sus rostros se delinea hacia el interior mismo y remite a la composición de elencos de gobierno y a la gestión, a los principios de jerarquización del ámbito académico y al movimiento estudiantil; el otro ubica a la universidad en relación con la esfera pública, interviniendo en la formulación de políticas públicas y en la construcción de vínculos con el campo político y cultural. Esta relación expresa un vínculo instituyente en un doble sentido: la sociedad interpela a la universidad y esta, a su vez, contribuye a la constitución de la sociedad de la que es parte. Desde esta perspectiva se pretende identificar e indagar las zonas de intersección entre la producción académica y la intervención cultural y política en el ámbito socio-político local y regional.

La pretensión de abordar el lapso de un siglo impone realizar precisiones de orden cronológico. Como es sabido, la UNL fue creada en 1919 en el marco general del proceso de modernización de la Argentina. Ya en su etapa fundacional se dio inicio a un conjunto de publicaciones institucionales $y$, a través del Instituto Social, a las primeras modalidades de extensión universitaria. Otro aspecto de la vida institucional estuvo dado por la conformación de los elencos de gobierno y de las diversas formaciones estudiantiles que contribuyeron a una cultura política reformista, afectados ambos por el golpe de Estado de 1943. A partir de ese momento se consideran cortes temporales siguiendo la periodización de la historia política argentina: la crisis del consenso liberal y los años del peronismo (1943-1955); los años sesenta, entre la caída del peronismo y el

\footnotetext{
1 El proyecto CAI+D fue aprobado por la Secretaría de Ciencia, Arte y Tecnología de la UNL en la convocatoria 2020. El grupo responsable está integrado por Fabiana Alonso (directora), Mariana Tettamanti (co-directora), Eliana Bertero y Juan Pablo Giordano. El grupo colaborador, por Pablo Salomon, Leonel Cescut y Máximo Deshayes.
} 
golpe de Estado de 1966; los primeros años setenta, desde el Cordobazo (1969) hasta el golpe de Estado de 1976; la última dictadura militar, autodenominada Proceso de Reorganización Nacional (1976-1983) y, finalmente, la reconstrucción democrática iniciada a fines de 1983.

\section{ESTADO DE LA CUESTIÓN Y MARCO TEÓRICO DE REFERENCIA}

En las últimas décadas, la investigación histórico-social ha configurado a la institución universitaria como objeto de estudio, en orden a indagar los procesos institucionales, políticos y culturales y las modalidades de producción de conocimiento (Halperin Donghi, 1962; Portantiero, 1978; Suasnábar, 2004; Graciano, 2008; Buchbinder, 2010).

Se entiende a la institución universitaria en general, y a la UNL en particular, como una figuración, esto es, un entramado de interdependencias entre individuos y grupos, sujeto a tensiones y reformulaciones en virtud de equilibrios de poder inestables (Elias, 2006), considerando al poder no como un atributo o una sustancia, sino en un sentido relacional. Esta perspectiva permite, a su vez, contemplar las complejas relaciones de la universidad con los cambiantes escenarios políticos de la Argentina del siglo XX. Por ello, se recuperan enfoques que permiten entender las prácticas de los agentes (individuos y grupos) sin descuidar las condiciones estructurales que condicionan y, a la vez, habilitan dichas prácticas.

En lo concerniente al proceso de construcción del objeto, se explicita a continuación la trama conceptual en la que se sustenta la investigación. Como parte integrante de la cultura —entendida como sistema significante a través del cual un orden social se comunica, se reproduce, se experimenta y se investiga (Williams, 1994)_, las prácticas desarrolladas por la UNL en su relación con la sociedad no se estudian como meras derivaciones del orden social sino como elementos activos en su constitución. Ubicándonos en ese registro, cobra importancia la indagación de representaciones, entendidas como el conjunto de ideas y acciones que dotan de sentido a las prácticas constructivas del mundo social (Chartier, 1992, 2000, 2007). La noción de representación habilita explorar las selecciones interesadas de aspectos significativos del pasado (desde los distintos 
presentes en la duración de un siglo) y cómo se han ido configurando memorias y tradiciones que combinan recuerdos y olvidos, cuyo análisis permite iluminar zonas opacas de la propia institución. En ese plano es relevante analizar los usos del pasado, esto es, las interpretaciones que la UNL ha realizado de su propio pasado y las representaciones del pasado provincial y nacional atravesadas por las distintas coyunturas políticas.

Dado que el núcleo del proyecto radica en el análisis de las prácticas de representación a través de los vínculos de la UNL con el ámbito socio-político local y regional, cabe señalar que el término práctica remite a las acciones de los agentes y de los grupos constituidos en el ámbito universitario, pero que no se circunscriben a dicho espacio sino que se proyectan en la esfera pública. En este sentido, las intervenciones ponderan lo que estudiantes y académicos tienen en común como intelectuales (Macor, 2009). El uso de este término refiere a quienes, en las sociedades modernas, detentan el monopolio de la producción de los bienes simbólicos (Bourdieu, 1999). El campo intelectual es el universo social que les es propio y, al igual que los otros campos que constituyen el espacio social, tiene una autonomía relativa, está regido por reglas que le son propias y atravesado por luchas en torno a la producción cultural legítima, conforme estrategias que dependen del lugar objetivo que los agentes ocupen en el mismo.

Para el caso argentino, hacia el Centenario es posible observar las primeras formas de articulación del campo intelectual a través de una serie de transformaciones que son indicadores de una profesionalización de la práctica de escribir (sobre todo por medio del periodismo), nuevos ámbitos de sociabilidad e instancias de consagración (Altamirano y Sarlo, 1997; Terán, 2008). Paralelamente, un incremento y una diversificación del público lector como consecuencia de la educación pública, acompañados de una expansión de la urbanización y de la industria cultural.

Al centrarnos en las relaciones de la UNL con la esfera pública, no se debe perder de vista que las intervenciones de los agentes (individuos y grupos) se realizan en tanto que intelectuales y actúan interrelacionados a través de círculos, publicaciones y movimientos que encuentran su lugar en el campo de la cultura y que, en ciertas circunstancias, se involucran en el debate cívico y político (Altamirano, 2013). Como ha sido puesto de relieve para el caso argentino, junto a la figura del intelectual puede contemplarse la del experto. Si los primeros detentan reconocimiento social por ejercer la cultura del discurso crítico (Gouldner, 1980), 
los expertos se definen desde la especialización y la calificación en el contexto de la sociedad industrial (Williams, 2008). Si bien diferenciadas, ambas figuras pueden combinarse y actuar en el espacio delimitado por el Estado, la academia, las empresas y el campo intelectual, entre los cuales se producen pasajes y se desarrollan distintas formas de intervención y legitimación (Neiburg y Plotkin, 2004).

\section{OBJETIVOS}

\section{GENERAL}

$>$ Contribuir al estudio de las relaciones de la UNL con el ámbito sociopolítico y cultural local y regional, por medio del análisis de prácticas significantes y de la intervención pública de los agentes universitarios.

\section{ESPECÍFICOS}

$>$ Reconstruir los aportes de los universitarios del Litoral en la formulación de políticas públicas de alcance local, regional y nacional.

$>$ Analizar la trama sociopolítica (local y regional) que condiciona y habilita la intervención de los agentes universitarios en las disputas por las tradiciones culturales, intelectuales y políticas.

$>$ Indagar la conformación de los elencos de gobierno de la UNL en coyunturas particularmente relevantes para la vida universitaria.

$>$ Identificar los espacios de configuración de representaciones, la circulación de las mismas y las formas de apropiación.

\section{ESTRATEGIA METODOLÓGICA}

El proyecto adopta una perspectiva de análisis que se nutre de diversas corrientes de la investigación histórica y social. En primer término se recuperan perspectivas relacionales — como las desarrolladas por Elias (2006), Bourdieu (2005) 
y Giddens (1995) — dado que permiten entender las prácticas de los agentes (individuos y grupos) sin descuidar las condiciones estructurales que habilitan y a la vez constriñen las acciones. En este sentido, se buscará analizar las estrategias y las representaciones sin perder de vista los principios de jerarquización y las posiciones objetivas de quienes actúan, evitando así el falso dilema entre objetivismo y subjetivismo.

Los aportes de la historia política renovada, de la historia conceptual, de la historia intelectual y del análisis del discurso resultan productivos en relación con la temática de investigación. A partir de la renovación que experimentó en los últimos treinta años, la historia política amplió su horizonte teórico y temático. Por un lado, el análisis de la política adquirió una relativa autonomía explicativa en relación a las otras dimensiones de la vida social y, por otro, adquirió relevancia el diálogo interdisciplinar de la historia con el resto de las ciencias sociales. El estudio del Estado y sus instituciones, un tema clásico, empezó a ser abordado desde una perspectiva que pone el acento en los problemas conceptuales y reduce la escala de observación, cobrando importancia los casos locales dado que su análisis permite complejizar la realidad nacional. Asimismo la relación sociedad política y sociedad civil se transformó en eje de atención y, por lo tanto, ganaron terreno los análisis sobre las formas de representación, la ciudadanía, el rol de las dirigencias y la sociabilidad (Sábato, 2007; Bohoslavsky y Soprano, 2010; Plotkin y Zimmermann, 2012).

Por su parte, la historia conceptual, al echar luz sobre el modo en que cobran sentido y se redefinen los conceptos políticos y sociales en la modernidad, posibilita plantear interrogantes sobre la utilización y los alcances de ciertos términos, las connotaciones, las convergencias y las disidencias que implican los usos de los mismos. Como se advierte en la obra de Koselleck $(1993,2001)$, si bien los conceptos cristalizan experiencias históricas, sufren alteraciones y adquieren nuevos significados. En la búsqueda de perspectivas afines, la investigación también se referencia en la historia intelectual que hoy constituye un vasto campo en el que convergen distintas orientaciones que abordan los hechos de discurso, y adjudican una importancia central al lenguaje para la comprensión histórica de las significaciones (Rosanvallon, 2003; Altamirano, 2005). Atenta a los procesos de producción, recepción y discusión de ideas, la historia intelectual permite analizar e interpretar tanto las representaciones simbólicas como los modos en 
que ellas se inscriben en la realidad social. Además de las ideas, este enfoque contempla la historia social y política de los agentes de la cultura y sus prácticas culturales (Altamirano, 2007; Jay, 2007; Tarcus, 2007).

En lo que refiere a la concepción del discurso como práctica social, el análisis del discurso se propone como una metodología interdisciplinaria para abordar las prácticas discursivas en las que se ponen en tensión las representaciones, las relaciones sociales y las relaciones de poder. Esto no significa entender que los hechos sociales se agotan en el discurso, sino que este es una vía de acceso para reconstruir las experiencias históricas. En este sentido, conviene considerar la observación de Skinner (2007) en cuanto a que los contextos intelectuales no se explican simplemente por un conjunto de determinaciones sociales, sino que reconstruirlos supone restituir las lecturas, los debates y las variaciones de significado. Para el análisis empírico se elaborarán corpus de fuentes a partir de las revistas universitarias, publicaciones de los centros de estudiantes, documentación oficial (legajos, resoluciones, actas de consejos, memorias institucionales), prensa periódica, ediciones de la UNL (libros, publicaciones institucionales, revistas, textos de divulgación), testimonios orales y archivos privados.

\section{BIBLIOGRAFÍA}

Altamirano, C. (2005). Para un programa de historia intelectual y otros ensayos. Buenos Aires: Siglo XXI.

Altamirano, C. (2007). Intelectuales. Notas de Investigación. Buenos Aires: Norma. Altamirano, C. (2013). Introducción general. En Myers, J. I. La ciudad letrada, de la conquista al modernismo. Buenos Aires: Katz Editores.

Bohoslavsky, E. - Soprano, Germán (2010). Un Estado con rostro humano. Funcionarios e instituciones estatales en Argentina (desde 1880 a la actualidad). Buenos Aires: Universidad Nacional del General Sarmiento y Prometeo Libros.

Bourdieu, P. (1999). Intelectuales, política y poder. Buenos Aires: Eudeba.

Bourdieu, P. (2005). La práctica de la sociología reflexiva. En Bourdieu, P. - Wacquant, L. Una invitación a la sociología reflexiva. Buenos Aires: Siglo XXI. 
Buchbinder P. (2010). Historia de las Universidades argentinas. Buenos Aires: Sudamericana.

Chartier, R. (1992). El mundo como representación. Historia cultural: entre práctica y representación. Barcelona: Gedisa.

Chartier, R. (2000). Las revoluciones de la cultura escrita. Diálogos e intervenciones. Barcleona: Gedisa.

Chartier, R. (2007). La historia o la lectura del tiempo. Barcelona: Gedisa.

Elias, N. (2006). Sociología fundamental. Barcelona: Gedisa.

Giddens, A. (1995). La constitución de la sociedad. Bases para una teoría de la estructuración. Buenos Aires: Amorrortu.

Gouldner, A. (1980). El futuro de los intelectuales y el ascenso de la nueva clase. Madrid, Alianza.

Graciano, O. (2008). Entre la torre de marfil y el compromiso político. Bernal: Universidad Nacional de Quilmes.

Halperin Donghi, T. (1962). La Universidad de Buenos Aires. Buenos Aires: Eudeba.

Jay, M. (2007). Pretensiones desvergonzadas. La historia intelectual como juicio del pasado. Prismas volumen 1, 153-157.

Koselleck, R. (1993). Futuro pasado. Para una semántica de los tiempos históricos. Barcelona: Paidós.

Koselleck, R. (2001). Los estratos del tiempo. Estudios sobre la historia. Barcelona: Paidós.

Macor, D. (2009). Tradición reformista y democracia. En 90 años haciendo historia, Universidad Nacional del Litoral 1919-2009. Edición Especial Aniversario.

Neiburg F. - Plotkin M. (comps.) (2004). Intelectuales y expertos. La constitución del conocimiento social en la Argentina. Buenos Aires: Paidós.

Plotkin, M. - Zimmermann, E. (2012). Los Saberes del Estado. Buenos Aires: Edhasa. Portantiero, J.C. (1978). Estudiantes y política en América Latina (1918-1938). EI proceso de la reforma universitaria. México: Siglo XXI.

Rosanvallon, P. (2003). Por una historia conceptual de lo político. Buenos Aires: FCE. Sabato, H. (2007). La política argentina en el siglo XIX: notas sobre una historia renovada. En Palacios, Guillermo (coord.). Ensayos sobre la nueva historia política de América Latina, siglo. XIX. México: El Colegio de México. 
Skinner, Q. (2007). Lenguaje, política e historia. Bernal: Universidad Nacional de Quilmes. Suasnábar, C. (2004). Universidad e intelectuales. Educación y política en la Argentina (1955-1976). Buenos Aires: FLACSO-Manantial.

Tarcus, H. (2007). Introducción. La historia intelectual y la problemática de la recepción. En Marx en la Argentina. Sus primeros lectores obreros, intelectuales y científicos, Buenos Aires: Siglo XXI.

Williams, R. (1994). Sociología de la cultura. Barcelona: Paidós.

Williams, R. (2008). Palabras clave. Un vocabulario de la cultura y la sociedad. Buenos Aires: Nueva Visión. 